\title{
Levantamento etnobotânico de plantas medicinais na cidade de Ipameri - GO
}

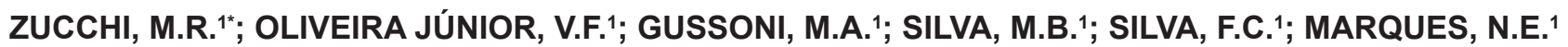
1 Unidade Universitária de Ipameri, Universidade Estadual de Goiás, Ipameri, GO, CEP: 75780-000. *e-mail: marceloribeiro.zucchi@gmail.com

RESUMO: Os objetivos deste trabalho foram: identificar as espécies vegetais utilizadas com fins medicinais pela comunidade de Ipameri (Estado de Goiás); investigar as preferências com relação à produção e comercialização dessas plantas; e diagnosticar o perfil de gênero e as faixas etárias e salariais de seus usuários. Para isso, foram realizadas entrevistas estruturadas com 200 famílias da cidade e coletadas as plantas visando-se a sua correta identificação. O material foi herborizado, identificado e depositado no Herbário da Universidade Estadual de Goiás (HUEG). Das 200 famílias entrevistadas, 75 disseram não fazer uso de plantas com fins medicinais (37,5\%), enquanto 125 afirmaram fazê-lo (62,5\%). O grupo que utiliza relacionou 35 espécies mais empregadas: hortelã-rasteira (Mentha $x$ villosa L.), boldo-setedores (Plectranthus barbatus Andrews.), capim-cidreira (Cymbopogon citratus (DC.) Stapf.), quebra-pedra (Phyllanthus niruri L.), camomila (Chamomilla recutita (L.) Rauschert.), poejo (Mentha pulegium L.), guaco (Mikania glomerata Spreng.), mentrasto (Ageratum conyzoides L.), alfavacão (Ocimum gratissimum L.), losna (Artemisia canphorata Vill.), bálsamo (Eysenhardtia platycarpa Mich.), carqueja (Baccharis trimera (Less.) DC.), funcho (Foeniculum vulgare Mill.), babosa (Aloe vera L.) e malva (Althaea officinalis L.). Todas as famílias consumidoras $(100 \%)$ afirmaram preferir as plantas cultivadas de forma orgânica, selecionando-as através da boa aparência (68\% das famílias) e consumindo-as in natura (sem beneficiamento, 100\%). A utilização de plantas medicinais em Ipameri é independente do sexo (54\%, mulheres e $46 \%$, homens) e se estende às várias faixas etárias e também sócio-econômicas, configurando-se assim, um bom mercado consumidor.

Palavras chaves: etnobotânica, plantas medicinais, policultivo

ABSTRACT: Ethnobotanical survey of medicinal plants in Ipameri City - Goiás State. The aims of this study were: to identify the plant species used for medicinal purposes by the community at Ipameri (Goiás State); to investigate the preferences with respect to the production and marketing of these plants; and to diagnose the gender profile and the age and wage ranges of users. Thus, structured interviews were conducted with 200 families in the city and plants were collected for their correct identification. The material was herborized, identified and deposited in the Herbarium of "Universidade Estadual de Goiás" (HUEG). Of the 200 families interviewed, 75 said they did not make use of plants for medicinal purposes (37.5\%), while 125 said they do use them (62.5\%). The latter group reported the 35 most used species: "hortelã-rasteira" (Mentha $x$ villosa L.), "boldo-sete-dores" (Plectranthus barbatus Andrews.), lemon grass (Cymbopogon citratus (DC.) Stapf.), "quebra-pedra" (Phyllanthus niruri L.), chamomile (Chamomilla recutita (L.) Rauschert.), pennyroyal (Mentha pulegium L.), guaco (Mikania glomerata Spreng.), mentrasto (Ageratum conyzoides L.), clove basil (Ocimum gratissimum L.), wormwood (Artemisia canphorata Vill.), balm (Eysenhardtia platycarpa Mich.), broom (Baccharis trimera (Less.) DC.), fennel (Foeniculum vulgare Mill.), aloe (Aloe vera L.) and mallow (Althaea officinalis L.). All consumer families (100\%) stated to prefer plants grown organically, to select the plants based on their good appearance (68\% families) and to eat them in natura (unprocessed, $100 \%$ ). The use of medicinal plants in Ipameri is independent of gender ( $54 \%$ womem and $46 \%$ men) and extends to several age and socioeconomic ranges, configuring thus a good consumer market.

Key words: ethnobotany, medicinal plants, polyculture

Recebido para publicação em 14/12/2009

Aceito para publicação em 17/12/2012

Rev. Bras. PI. Med., Campinas, v.15, n.2, p.273-279, 2013. 


\section{INTRODUÇÃO}

A Etnobotânica inclui estudos concernentes ao relacionamento mútuo entre populações tradicionais e plantas (Cotton, 1996). Esta ligação tem ocorrido desde que o homem iniciou o uso dos vegetais, para satisfazer suas necessidades de sobrevivência, seja como alimento, para produzir calor, para abrigar-se, na construção, como ornamento ou para assegurar sua saúde (Levy \& Aguirre, 1999).

Segundo Albuquerque (2000), a Etnobotânica faz a mediação dos variados discursos culturais, almejando a compreensão do outro, do seu modo de vida, dos seus códigos e costumes que racionalizam suas relações com a natureza. Então, a Etnobotânica tem grande importância para as populações regionais no que toca à exploração e manejo de recursos para obtenção de remédios, alimentos e matérias-primas (Ferro, 2006).

Os estudos etnobotânicos são muito importantes no Brasil, já que seu território abriga uma das mais ricas floras do mundo, no entanto, apenas $0,4 \%$ são quimicamente conhecidas (Gotlieb et al., 1996). Em sua complexa biodiversidade, existe grande número de plantas que são utilizadas pelas populações para o tratamento de diversas enfermidades, tanto para seres humanos quanto para animais domésticos (Ming, 1995).

Nesse contexto, o bioma Cerrado é um complexo vegetacional que detém grande parte dessa diversidade biológica (cerca de 235.000 espécies de angiospermas), e que ocupa extensa área territorial (estimada em 1,8 milhão $\mathrm{Km}^{2}$ ) nas regiões centrais do Brasil, as quais apresentam grande concentração populacional, o que justifica a importância deste tipo de estudo (Vila Verde et al., 2003). O município de Ipameri, no Estado de Goiás, está inserido nesse bioma.

Para efeito deste estudo, planta medicinal é todo vegetal que contém, em um ou vários de seus órgãos, princípios ativos que podem ser empregados para objetivos terapêuticos ou precursores de substâncias utilizadas para tais fins, sendo amplamente aplicadas pela medicina alternativa (Amorozo, 2002). Provavelmente, o uso das plantas no controle de enfermidades seja tão antigo quanto o homem. Há cerca de 3000 anos antes de Cristo, os chineses já utilizavam e cultivavam ervas medicinais, que hoje ainda são usadas com eficácia tanto na medicina popular, como por laboratórios de produtos farmacêuticos (Rodrigues et al., 2001). A utilização de plantas para o tratamento da saúde permanece até os dias de hoje fazendo parte da cultura de diferentes comunidades populacionais (Marondin, 2001).

De acordo com Rodrigues (1998), tendo em vista a importância de sanar, ou pelo menos de amenizar os problemas socioeconômicos da população brasileira, é crescente a preocupação em se estabelecer grupos de plantas medicinais para pesquisas, suas potencialidades, usos e meios de conservação desses recursos genéticos. Atualmente, o cultivo das ervas medicinais é um processo muito importante para a conservação das espécies vegetais, porque a retirada de plantas nativas de seu ambiente natural tem levado, em muitos casos, à redução drástica das populações destas espécies (Reis et al., 2003).

Para Ming et al. (2003), os pequenos produtores apresentam o perfil adequado para cultivar plantas medicinais, que são orgânicas na sua essência e não permitem o plantio em larga escala, exigindo o policultivo como forma de proteger as espécies de enfermidades e pragas. Portanto, os pequenos produtores podem encontrar na produção de espécies medicinais uma oportunidade ímpar de diversificar suas propriedades e aumentar sua renda.

Entretanto, para se obter sucesso neste tipo de empreendimento, deve-se ter o domínio das técnicas de cultivo e conhecer a etapa de comercialização dos produtos. O desconhecimento do mercado consumidor pode causar remuneração inferior ou mesmo prejuízo por ocasião da venda (Junqueira \& Luengo, 2000). Segundo Vilela \& Macedo (2000), as tendências de mercado devem ser observadas atentamente, para identificar quais os atributos dos bens (neste caso, as plantas medicinais) que têm maior valor para os consumidores, e assim aproveitar as reais oportunidades de mercado.

Neste contexto, a etnobotânica documenta o uso das plantas medicinais devido à sua aplicação farmacológica potencial. Do mesmo modo, busca contribuir com o uso sustentável dos recursos naturais associados a esta atividade, e quantificá-los e inventariá-los é um primeiro passo para alcançar este objetivo (Tuxill \& Nabhan, 1998; Martin, 2000). Nesse sentido, os objetivos deste trabalho foram: identificar as espécies vegetais utilizadas com fins medicinais pela comunidade de Ipameri (Estado de Goiás); investigar as preferências com relação à produção e comercialização dessas plantas; e diagnosticar o perfil de gênero e as faixas etárias e salariais dos seus usuários.

\section{MATERIAL E MÉTODO}

Esta pesquisa foi realizada no período de agosto de 2008 a junho de 2009, na cidade de Ipameri, localizada na região Sul do Estado 
de Goiás, na micro-região denominada Estrada de Ferro ou Sudeste Goiano. Ipameri limita-se ao Norte com os municípios de Cristalina e Luziânia; a Noroeste, com Orizona e Urutaí; a Oeste, com Pires do Rio e Caldas Novas; a Sudoeste, com Corumbaíba; ao Sul, com Goiandira e Nova Aurora; a Leste, com Catalão; e a Nordeste, com Campo Alegre de Goiás, parte de Cristalina e Paracatu. A área do município é de $4.691 \mathrm{~km}^{2}$, com 23.014 habitantes. A escolha desse município para esta pesquisa foi baseada em sua história e cultura, pois trata-se de uma cidade bastante antiga, com forte vocação agrícola e com uma população nativa tradicional que ainda hoje pratica costumes que foram herdados de seus antepassados.

Para a investigação sobre a utilização, as preferências com relação à produção e comercialização das plantas medicinais, e o perfil etário e sócio-econômico dos usuários dessas plantas, foram aplicados 200 questionários na forma de entrevistas estruturadas e listagem livre das plantas medicinais, em 200 residências (famílias), totalizando-se 680 pessoas. O método de amostragem utilizado foi probabilístico, com as residências incluídas ao acaso. Para cada bairro foram selecionadas, em ruas diferentes, 05 residências. Deste modo, objetivou-se amostrar as diferentes regiões da cidade. Somente as pessoas que moram efetivamente na casa, foram consideradas como membros da família, conforme procedimento de Rodrigues \& Guedes (2006). No momento das entrevistas, quando havia mais de uma pessoa da família, essas às vezes participaram das respostas ao questionário. Este foi baseado nas metodologias de Alexiades (1996), Martin (2000), Rodrigues \& Carvalho (2001), Marchese et al. (2004) e Albuquerque et al. (2008), para caracterizar diferentes aspectos da comunidade e dos usuais consumidores das plantas.

As entrevistas foram realizadas por acadêmicos dos cursos de Agronomia e Engenharia Florestal da Universidade Estadual de Goiás, Unidade Universitária de Ipameri. Após cada entrevista, foram coletadas as plantas que estavam em estágio reprodutivo visando a sua identificação. O material botânico coletado foi herborizado segundo os métodos usuais e depositado no Herbário da Universidade Estadual de Goiás (HUEG), na Unidade Universitária de Ciências Exatas e Tecnológicas (UnUCET / UEG). A identificação taxonômica foi feita de acordo com suas características morfológicas vegetativas e reprodutivas, utilizando Lorenzi \& Matos (2008), Almassy Júnior et al. (2005) e Martins et al. (2000) ou comparando com exsicatas de herbários. As origens das plantas estudadas foram verificadas nas mesmas referências usadas para a identificação.

\section{RESULTADO E DISCUSSÃO}

Das 200 famílias entrevistadas, 75 disseram que não utilizam plantas com fins medicinais (37,5\%), enquanto 125 afirmaram fazê-lo (62,5\%). Estas fazem uso de uma quantidade diversificada de plantas (35 espécies distribuídas em 30 gêneros e em 14 famílias), destacando-se por serem as mais utilizadas: hortelã-rasteira (Mentha $x$ villosa L.), boldo-sete-dores (Plectranthus barbatus Andrews.), capim-cidreira (Cymbopogon citratus (DC.) Stapf.), quebra-pedra (Phyllanthus niruri L.), camomila (Chamomilla recutita (L.) Rauschert.), poejo (Mentha pulegium L.), guaco (Mikania glomerata Spreng.), mentrasto (Ageratum conyzoides L.), alfavacão (Ocimum gratissimum L.), losna (Artemisia canphorata Vill.), bálsamo (Eysenhardtia platycarpa Mich.), carqueja (Baccharis trimera (Less.) DC.), funcho (Foeniculum vulgare Mill.), babosa (Aloe vera L.) e malva (Althaea officinalis L.)(Tabela 1).

Souza \& Felfili (2006) observaram que na região do Alto Paraíso de Goiás (GO), as famílias de plantas medicinais mais usadas foram Asteraceae (com 14 espécies) e Fabaceae (com 11 espécies). Esses dados são um pouco diferentes deste trabalho, em que predominaram as famílias Lamiaceae (com 10 espécies) e Asteraceae (com 08 espécies, Tabela 1). Estes resultados são mais parecidos com os de Jacomassi \& Piedade (1994), os quais observaram que as famílias com mais plantas medicinais utilizadas pela população de Goioerê (PR) foram Asteraceae com 09 espécies e Lamiaceae com 06 espécies.

Coe \& Anderson (1999) citado por Pasa et al. (2006), em estudo etnobotânico realizado na Nicarágua, observaram que a maioria da população pesquisada mantém ao redor de suas casas algumas plantas de uso medicinal, e o mesmo aconteceu na região da Vila Canaã em Goiânia (GO), em trabalho realizado por Silva \& Souza (2007), dados que são corroborados com a presente pesquisa, na qual 76 residências amostradas tinham plantas medicinais em seus quintais.

Neste trabalho foi verificada a presença de cinco "curandeiros", pessoas mais velhas (acima de 60 anos) bastante procuradas por conhecerem e cultivarem plantas com fins medicinais, assim como ocorreu na Região do Alto do Rio Grande em Minas Gerais, em trabalho conduzido por Rodrigues \& Carvalho (2001).

A enfermidade que mais ocasiona o uso de várias plantas citadas nesta pesquisa é a gripe $(35 \%)$, seguida por transtornos digestivos $(18 \%)$. Os problemas renais foram mencionados por $6 \%$, enquanto as dores-de-cabeça e os transtornos neurológicos foram ditos por $5 \%$ das famílias, cada um desses distúrbios. As famílias restantes (31\%) disseram usar as plantas para outras variadas

Rev. Bras. PI. Med., Campinas, v.15, n.2, p.273-279, 2013. 
TABELA 1. Plantas medicinais utilizadas pela população de Ipameri (Goiás), organizadas em famílias, nomes científicos, nomes populares e suas origens. Todas as espécies que foram depositadas em herbário estão acompanhadas de seu número de registro.

\begin{tabular}{|c|c|c|c|}
\hline Família / Nome Científico & Nome Comum & Origem & Número de registro \\
\hline \multicolumn{4}{|l|}{ Amaranthaceae } \\
\hline Alternanthera dentata (Moench.) Stuchlik & Terramicina & Brasil & 6981 \\
\hline Chenopodium ambrosioides L. & Erva-de-santa-maria & América Tropical & 6980 \\
\hline \multicolumn{4}{|l|}{ Apiaceae } \\
\hline Foeniculum vulgare Mill. & Funcho & Mediterrâneo & 6982 \\
\hline \multicolumn{4}{|l|}{ Asphodelaceae } \\
\hline Aloe vera $\mathrm{L}$. & Babosa & Sul da África & - \\
\hline \multicolumn{4}{|l|}{ Asteraceae } \\
\hline Ageratum conyzoides $\mathrm{L}$. & Mentrasto & Brasil & 6970 \\
\hline Artemisia canphorata Vill. & Losna & Ásia e Europa & 6971 \\
\hline Baccharis trimera (Less.) D.C. & Carqueja & América do Sul & 6972 \\
\hline Chamomilla recutita (L.) Rauschert. & Camomila & Europa & 6969 \\
\hline Mikania glomerata Spreng. & Guaco & América do Sul & - \\
\hline Solidago chilensis Meyen. & Arnica & América do Sul & - \\
\hline Vernonia condensata Baker. & Boldo, Boldo-chinês & África & 6974 \\
\hline \multicolumn{4}{|l|}{ Asteraceae } \\
\hline Vernonia polyanthes Less. & Assa-peixe & Brasil & 6973 \\
\hline \multicolumn{4}{|l|}{ Fabaceae } \\
\hline Myroxylon peruiferum L. f. & Bálsamo & América do Sul & - \\
\hline \multicolumn{4}{|l|}{ Lamiaceae } \\
\hline Lavandula angustifolia Mill. & Alfazema & Europa & 6968 \\
\hline Melissa officinalis L. & Erva-cidreira, Melissa & Europa e Ásia & - \\
\hline Mentha arvensis L. & Vique & Japão & - \\
\hline \multirow[t]{2}{*}{ Mentha pulegium $\mathrm{L}$. } & Poejo & Sul do Brasil, & \\
\hline & & Uruguai e Argentina & 6965 \\
\hline Mentha x villosa L. & Hortelã-rasteira & Ásia & 6963 \\
\hline Ocimum gratissimum $\mathrm{L}$. & Alfavacão & América do Sul & 6966 \\
\hline Plectranthus amboinicus (Lour.) Spreng. & Hortelã-da-folha-gorda & Nova Guiné & - \\
\hline \multirow[t]{2}{*}{ Plectranthus barbatus Andrews. } & Boldo-sete-dores, & & \\
\hline & Falso-boldo & África Tropical e Índia & 6964 \\
\hline Rosmarinus officinalis L. & Alecrim & Mediterrâneo & 6967 \\
\hline Salvia officinalis L. & Sálvia & Mediterrâneo & - \\
\hline \multicolumn{4}{|l|}{ Lauraceae } \\
\hline Cinnamomum canphora Turra & Cânfora & Brasil & - \\
\hline Cinnamomum zeylanicum Breyn. & Canela & Índia & 6975 \\
\hline
\end{tabular}


TABELA 1. Plantas medicinais utilizadas pela população de Ipameri (Goiás), organizadas em famílias, nomes científicos, nomes populares e suas origens. Todas as espécies que foram depositadas em herbário estão acompanhadas de seu número de registro.

continuação...

\begin{tabular}{|c|c|c|c|}
\hline Família / Nome Científico & Nome Comum & Origem & Número de registro \\
\hline \multicolumn{4}{|l|}{ Malvaceae } \\
\hline Gossypium hirsutum L. & Algodão & América & 6976 \\
\hline Malva parviflora $\mathrm{L}$. & Malva & Brasil & - \\
\hline \multicolumn{4}{|l|}{ Phyllanthaceae } \\
\hline Phyllanthus niruri L. & Quebra-pedra & Brasil & 6983 \\
\hline \multicolumn{4}{|l|}{ Phytolaccaceae } \\
\hline Petiveria alliacea L. & Guiné & América Tropical & 6984 \\
\hline \multicolumn{4}{|l|}{ Plantaginaceae } \\
\hline Plantago major L. & Tanchagem & Europa & 6985 \\
\hline \multicolumn{4}{|l|}{ Poaceae } \\
\hline \multirow[t]{2}{*}{ Cymbopogon citratus (DC.) Stapf. } & Capim-cidreira, & & \\
\hline & Capim-limão & Índia & 6986 \\
\hline \multicolumn{4}{|l|}{ Rutaceae } \\
\hline Citrus limon L. & Limão & Ásia & - \\
\hline Ruta graveolens L. & Arruda & Mediterrâneo & 6977 \\
\hline \multicolumn{4}{|l|}{ Zingiberaceae } \\
\hline Curcuma longa L. & Açafrão & Índia & 6978 \\
\hline Zingiber officinale Roscoe & Gengibre & Ásia Tropical & 6979 \\
\hline
\end{tabular}

doenças (sem especificá-las).

A parte das plantas mais utilizada como remédio é a folha (citada por $40 \%$ das famílias). 0 uso da planta toda foi afirmado por $28 \%$. Remédios em que são usadas as flores foram mencionados por $19 \%$. O uso das raízes por $8 \%$ e o uso das cascas das plantas por apenas $5 \%$ das famílias. De modo similar, o largo uso das folhas na preparação dos remédios também foi detectado por Jacomassi \& Piedade (1994). Os remédios, em sua maioria, são preparados na forma de chá (infusão ou decocto) ou xarope, variando de acordo com as espécies e os objetivos de tratamento, em geral preparados pelos próprios usuários.

Algumas plantas medicinais exigem, segundo os especialistas, cuidados especiais em sua administração. Tais cuidados podem estar diretamente relacionados à planta ou à maneira como o remédio foi preparado (Calixto \& Ribeiro, 2002). Quanto às formas de preparo e utilização desses remédios caseiros, percebe-se que eles podem ser usados, conforme o caso, como preparações diversas (chá infuso, decoctos, maceração, etc.) para uso interno e, também, outros tipos de preparações (cataplasma, compressa, unguento, etc.) para o uso externo.

De acordo com Martins et al. (2000), as formas mais comumente usadas nos tratamentos caseiros com plantas medicinais são as seguintes: aluá, cataplasma, chás, inalação, xarope e vinho medicinal. Aluá é uma bebida praticamente fermentada feita com raízes. Cataplasma é uma preparação com farinha, água e a planta triturada (para uso externo). Os chás podem ser feitos por infusão, por cozimento ou por maceração. A inalação combina a ação do vapor de água quente com o aroma das plantas medicinais. No xarope juntase parte do chá com uma parte de açúcar do tipo cristalizado, obtendo-se assim o xarope frio que deve ser filtrado após três dias de contato. E no vinho medicinal, a planta é deixada em maceração durante oito dias com vinho tinto, gerando uma 
bebida estimulante. Neste estudo predominaram as preparações como chás (por decocção ou por infusão) e os xaropes, mas algumas famílias também relataram o uso de cataplasmas.

Assim como foi constatado por Víglio (2000) no Rio de Janeiro, as famílias ipamerinas consumidoras de plantas medicinais também as preferem cultivadas de forma orgânica (100\%), e selecionando as melhores através da boa aparência (68\%). Outras (29\%) as escolhem pela sua procedência, e as restantes $(03 \%)$ selecionamnas por outros motivos. Preferencialmente, todas consomem as ervas in natura, ou seja, sem beneficiamento, afirmando que o consumo de plantas beneficiadas (adquiridas em supermercados ou frutarias) ocorre apenas na indisponibilidade de alguma delas em seus quintais.

Também foi verificado que nas famílias que utilizam as ervas medicinais, tanto as mulheres (54\%) quanto os homens (46\%) o fazem na mesma intensidade, com raras exceções, assim como ocorreu em estudo realizado por Cerveira \& Castro (1999) na cidade de São Paulo. A faixa etária dos consumidores de plantas medicinais também foi bastante variável, visto que nestas famílias quase todos os membros são consumidores. Entretanto, a frequência de utilização dessas plantas é muito variável, dependendo do estado de saúde dos integrantes da família, sendo geralmente diária em caso de alguma enfermidade, ou uma vez por semana quando não há problemas de saúde.

Esta pesquisa também detectou a importância da transmissão oral dos conhecimentos de geração para geração, pois a maioria das famílias consumidoras $(90 \%)$ afirmou ter aprendido com parentes mais velhos, como avós, ou até mesmo com "benzedores", sobre a importância das plantas medicinais e as formas de preparo destas. Essa transferência de conhecimentos de geração para geração já foi notada por Albuquerque (1999) em estudo realizado com descendentes culturais do africano no Brasil. De acordo com Medeiros et al. (2004), os meios modernos de comunicação causam perda dessa transmissão oral dos conhecimentos sobre os usos das plantas, mas isso não ocorreu na população ipamerina, como no exemplo citado de Albuquerque (1999) e também em Rodrigues \& Guedes (2006).

Com relação ao quadro sócio-econômico da população ipamerina, revelou-se bastante heterogêneo, já que o rendimento mensal das famílias é bastante variável, desde menos de um até oito salários mínimos. Entretanto, em qualquer situação de renda, encontram-se famílias consumidoras de algum tipo de planta medicinal. O questionário aplicado nas entrevistas tinha uma pergunta sobre quais as plantas eram mais usadas pela família, e a média das respostas foi 3,4 plantas por família entrevistada. Esse uso distribuído em todos os níveis de rendimentos também ocorreu no estudo realizado por Vendruscolo (2005), em Porto Alegre, Rio Grande do Sul.

Deste modo, os pequenos produtores locais poderiam produzir aquelas plantas medicinais indisponíveis ou com menor disponibilidade nos quintais das residências. Esta seria uma possibilidade de rendimento extra, ampliando e diversificando o orçamento mensal desses produtores. Segundo Naiverth \& Faria (2007), existe uma tendência de produção dessas plantas por pequenos agricultores, sendo uma boa alternativa a formação de pequenas cooperativas, aliando-se os conhecimentos empíricos diversos e também plantas diferentes.

Com base nos resultados desta pesquisa, pôde-se chegar a algumas conclusões. A comunidade de Ipameri faz um uso diversificado de plantas medicinais (35 espécies identificadas em 30 gêneros e em 14 famílias botânicas). Em caso de interesse na comercialização dessas plantas, as mesmas deverão ser cultivadas de maneira orgânica, mantidas na forma in natura, e com um cuidado especial com a aparência. A utilização de plantas medicinais em Ipameri é independente do sexo e se estende às várias faixas etárias e também sócio-econômicas, representando, portanto, um bom mercado consumidor.

\section{AGRADECIMENTO}

Os autores agradecem à Pró-Reitoria de Pesquisa da Universidade Estadual de Goiás pela bolsa de iniciação científica concedida ao acadêmico Valtair Fernandes de Oliveira Júnior para o desenvolvimento deste trabalho. E agradecem também aos revisores pelas críticas e sugestões.

\section{REFERÊNCIA}

ALBUQUERQUE, U.P. Referências para o estudo da etnobotânica dos descendentes culturais do africano no Brasil. Acta Farmacéutica Bonaerense, v.18, n.4, p.299-306, 1999.

ALBUQUERQUE, U.P. A etnobotânica no nordeste brasileiro. In: CAVALCANTI, T.B. \& WALTER, B.M.T. Tópicos Atuais em Botânica: Palestras Convidadas do $51^{\circ}$ Congresso Nacional de Botânica. Brasília: Embrapa Recursos Genéticos e Biotecnologia / Sociedade Botânica do Brasil, 2000. p.241-249.

ALBUQUERQUE, U.P.; LUCENA, R.F.P; CUNHA, L.V.F.C. Métodos e técnicas na pesquisa etnobotânica. $2^{\mathrm{a}} \mathrm{Ed}$. Recife: Comunigraf, 2008. 323p.

ALEXIADES, M.N. Collecting ethnobotanical data: an introduction to basic concepts and techniques. In: (Org.). Selected guidelines for ethnobotanical research: a field manual. New York: NYBG, 1996. p.53-94. 
ALMASSY JÚNIOR, A.A.; LOPES, R.C.; ARMOND, C.; SILVA, F.; CASALI, V.W.D. Folhas de Chá - Plantas Medicinais na Terapêutica Humana. Viçosa: UFV, 2005. 233p.

AMOROZO, M.C.M. Uso e diversidade de plantas medicinais em Santo Antônio do Leverger, MT, Brasil. Acta Botanica Brasilica, v.16, n.2, p.189-203, 2002.

CALIXTO, J.S.; RIBEIRO, E.M. O Cerrado como fonte de plantas medicinais para uso dos moradores de comunidades tradicionais do Alto Jequitinhonha, MG. 2002. Monografia (Graduação em Engenharia Florestal) - Universidade Federal de Lavras, Lavras.

CERVEIRA, R.; CASTRO, M.C. Consumidores de produtos orgânicos da cidade de São Paulo: características de um padrão de consumo. Informações Econômicas, v.29, n.12, p.7-20, 1999.

COE, F.G.; ANDERSON, G.J. Ethnobotany of the Sumu (Ulwa) of Southeastern Nicaragua and comparisons with Miskitu plant lore. Economic Botany, v.53, n.4, p.364-394, 1999.

COTTON, C.M. Ethnobotany: principles and applications. New York: J. Wiley, 1996. 320p.

FERRO, D. Fitoterapia: conceitos clínicos. São Paulo: Atheneu, 2006. 502p.

GOTTLIEB, O.R.; KAPLAN, M.A.C.; BORIM, M.R.M.B. Biodiversidade. Um enfoque químico - biológico. Rio de Janeiro: Editora da UFRJ, 1996. 267p.

JACOMASSI, E.; PIEDADE, L.H. A importância das plantas com finalidades terapêuticas e suas aplicações na cidade de Goioerê-PR. Revista UNIMAR, v.16, n.2, p.335-353, 1994.

JUNQUEIRA, A.H.; LUENGO, R.F.A. Mercados diferenciados de hortaliças. Horticultura Brasileira, v.18, n.2, p.95-99, 2000.

LORENZI, H.; MATOS, F.J.A. Plantas Medicinais no Brasil: nativas e exóticas cultivadas. $2^{\mathrm{a}} \mathrm{ed}$. Nova Odessa: Instituto Plantarum, 2008. 564p.

MARCHESE, J.A.; MING, L.C.; GALINA, A.; TEDESCO, A.C.; CONTE, C.; MINIUK, C.M.; SCHURT, D.A.; SANGALETTI, E.; DA SILVA, G.O.; GOMES, G.; BERTAGNOLLI, J.A.; DE FRANCHESCHI, L.; COSSA, M.L.; DE MORAES, M.R.D.; DE LIMA, P.M.; LIRA, R.; CAMOCHENA R.; COSTA, S. Popular use of the medicinal plants for the rural community of the Passo da Ilha, in the Pato Branco city, Paraná State, Brazil. In: THE 3RD WORLD CONGRESS ON MEDICINAL AND AROMATIC PLANTS FOR HUMAN WELFARE - WOCMAP, 2003, Chiang Mai. Proceedings of the 3rd WOCMAP. Chiang Mai - Tailândia, 2003. p.07-07.

MARTIN, G.J. Etnobotánica - Manual de métodos: manuales de conservación. Série Pueblos y Plantas 1, WWF. Uruguay: Ed. Nordan-Comunidad, 2000. 268p.

MARTINS, E.R.; CASTRO,D.M.; CASTELLANI, D.C.; DIAS, J.E. Plantas Medicinais. Viçosa: UFV, 2000. 226p.

MEDEIROS, M.F.T.; FONSECA, V.S.; ANDREATA, R.H.P. Plantas medicinais e seus usos pelos sitiantes da Reserva Rio das Pedras, Mangaratiba, RJ, Brasil. Acta Botanica Brasilica, v.18, n.2, p.391-399, 2004.

MING, L.C. Levantamento das plantas medicinais na Reserva Extrativista Chico Mendes, Acre. 1995. 175p. Tese (Doutorado) - Universidade Estadual Paulista, Botucatu.

MING, L.C.; SILVA, S.M.P.; SILVA, M.A.S.; HIDALGO,
A.F.; MARCHESE, J.A.; CHAVES, F.C.M. Manejo e cultivo de plantas medicinais: algumas reflexões sobre perspectivas e necessidades no Brasil. In: Diversos olhares em etnobiologia, etnoecologia e plantas medicinais. Cuiabá: Unicen, 2003. p.149-156.

NAIVERTH, J.A.; FARIA, C.M.D. Cultivo de Plantas Medicinais como Alternativa de Renda para Agricultores Familiares do Município de Candói - PR. Revista Eletrônica Lato Sensu, p.27-32, 2007. Curitiba: julho, 2007. Ano 2, n.1, ISSN 1980-6116. Disponível em: http://www.unicentro.br - Ciências Agrárias. Acesso em: 18 jun. 2009.

PASA, M.C.; SOARES, J.J.; GUARIM NETO, G. Estudo etnobotânico na comunidade de Conceição-Açu. Acta Botanica Brasilica, v.19, n.2, p.195-207, 2006.

REIS, M.S.; MARIOT, A.; STEENBOCK, W. Diversidade e domesticação de plantas medicinais. In: SIMÕES, C.M.O.; SCHENKEL, E.P.; GOSMAN, G.; MELLO, J.C.P.; MENTZ, L.A.; PETROVICK, P.R. (Ed.). Farmacognosia: da planta ao medicamento. $5^{\mathrm{a}} \mathrm{ed}$. Porto Alegre/Florianópolis: Editora da UFRGS/Editora da UFSC, 2003. p.45-74.

RODRIGUES, A.C.C.; GUEDES, M.L.S. Utilização de plantas medicinais no Povoado Sapucaia, Cruz das Almas - Bahia. Revista Brasileira de Plantas Medicinais, v.8, n.2, p.1-7, 2006.

RODRIGUES, V.E.G. Levantamento florístico e etnobotânico de plantas medicinais dos cerrados na região do Alto Rio Grande - Minas Gerais. 1998. 235p. Dissertação (Mestrado em Engenharia Florestal) - Universidade Federal de Lavras, Lavras.

RODRIGUES, V.E.G.; CARVALHO, D.A. de. Levantamento etnobotânico de plantas medicinais do domínio cerrado na região do Alto Rio Grande - Minas Gerais. Ciência Agrotécnica, v.25, n.1, p.102-123, 2001.

RODRIGUES, V.E.G.; CARVALHO, D.A. Plantas medicinais no domínio dos cerrados. Lavras: Editora UFLA, 2001. 180p.

SILVA, J.O.; SOUZA, P.S. Levantamento etnobotânico das plantas medicinais utilizadas pela população da Vila Canaã, região sudeste, Goiânia, Goiás. Ciência Agrotécnica, v.32, p.87-88, 2007.

SOUZA, C.D.; FELFILI, J.M. Uso de plantas medicinais na região de Alto Paraíso de Goiás, GO. Acta Botanica Brasilica, v.20, n.1, p.135-142, 2005.

TUXILL, J.; NABHAN, G.P. Plantas, comunidades y áreas protegidas: una guía del manejo in situ. Fundo Mundial para La Naturaleza - WWF, 1998.

VENDRUSCOLO, G.S.; RATES, S.M.K.; MENTZ, L.A. Dados químicos e farmacológicos sobre as plantas utilizadas como medicinais pela comunidade do bairro Ponta Grossa, Porto Alegre, Rio Grande do Sul. Revista Brasileira de Farmacognosia, v.15, p.361-372, 2005.

VÍGLIO, E.C.B.L. Produtos orgânicos: uma tendência para o futuro ? Agroanalysis, v.16, n.12, p.8-11, 1996.

VILA VERDE, G.M.; PAULA, J.R.; CARNEIRO, D.M. Levantamento etnobotânico das plantas medicinais do cerrado utilizadas pela população de Mossâmedes (GO). Revista Brasileira de Farmacognosia, v.13, p. 64-66, 2003.

VILELA, N.J.; MACEDO, M.M.C. Fluxo de poder no agronegócio: o caso das hortaliças. Horticultura Brasileira, v.18, n.2, p.88-94, 2000. 